\title{
How best to determine causative pathogens of pneumonia
}

\author{
David R. Murdoch
}

\begin{abstract}
The biggest recent development in pneumonia diagnostics has been the increased availability and use of nucleic acid detection assays, although this change has brought with it new challenges about the interpretation of positive results. Recognition of the existence of the lung microbiome has challenged the traditional views of pneumonia pathogenesis and may provide the opportunity for new diagnostic tools that are focused on more than just detection of specific known pathogens.
\end{abstract}

Keywords: Pneumonia, Diagnostics, Aetiology, Polymerase chain reaction, PCR

In 2009, some colleagues and I wrote a commentary on pneumonia diagnostics [1]. This is a challenging area. Historically, even with the best of methods, we have been unable to define a causative pathogen in a significant proportion of pneumonia episodes, especially in children. The impact of suboptimal diagnostics extends beyond individual patient care, also limiting our ability to assess interventions such as vaccines. Timed to coincide with the first World Pneumonia Day, our commentary tried to encourage a fresh look at pneumonia diagnostics. How has the world changed in the past seven years?

In 2009, we were still reliant on traditional diagnostics based on microscopy and culture-methods that had been around for decades. There had been modest advances in antigen detection methods, limited mainly to pneumococcal disease in adults and Legionnaires' disease. Nucleic acid detection tests (NATs), such as polymerase chain reaction (PCR), had been established for all major pneumonia pathogens and were the single biggest development in the area, although widespread uptake by diagnostic laboratories had been slow. We highlighted several issues that needed addressing in order to move forward. The inability to obtain good quality specimens from the lower respiratory tract was a fundamental problem. Many diagnostic methods, such as blood culture, had poor diagnostic sensitivity, while the assessment of diagnostic accuracy

Correspondence: david.murdoch@otago.ac.nz

Department of Pathology, University of Otago, Christchurch, P.O. Box 4345, Christchurch 8140, New Zealand was made challenging due to the lack of suitable comparator ("gold") standards. Importantly, assigning causation was a struggle. Many pneumonia pathogens can also be asymptomatic colonisers of the upper respiratory tract, so distinguishing innocent bystanders from true pathogens can be challenging when detected in clinical specimens from non-sterile sites, such as expectorated sputum. We emphasised the need to look beyond technology, and the importance of assessing diagnostic performance within clinical and epidemiologic contexts. We were also hopeful for innovative new approaches.

So, what does the situation look like in 2016? A major change for diagnostic laboratories over the past seven years has been the increased availability of commercial NATs for respiratory pathogens. There are now many choices, typically in multiplex format, and the range continues to expand. Indeed, debate has started about whether these large multiplex panels should be subjected to restricted use in order to ensure appropriate use [2]. Furthermore, the successful deployment around the world of the Xpert ${ }^{\circ}$ MTB/RIF assay (Cepheid, United States of America) for tuberculosis diagnostics has demonstrated the potential use of NATs beyond well-equipped laboratories and into resource-limited settings [3]. Despite their increased availability, NATs do not yet have clearly established roles for some major pneumonia pathogens. In particular, the inability to reliably distinguish Streptococcus pneumoniae carriage from disease when testing respiratory specimens is a major limitation, although setting thresholds based on bacterial load has shown promise in 
adults with pneumonia [4]. While used in some surveillance programmes [5], PCR testing of blood specimens for $S$. pneumoniae is not an established test for diagnosing pneumococcal pneumonia in individual patients due to uncertainty about diagnostic accuracy across different populations.

Therefore, in the context of pneumonia, NATs are still mainly useful for detection of non-colonising bacteria, such as Legionella species [6] and Mycobacterium tuberculosis [3], and for respiratory viruses. Even then, casecontrol studies have demonstrated the need for caution with the interpretation of respiratory virus test results, as positive results have been found to be as common in healthy controls as among pneumonia patients, especially for viruses other than influenza viruses, respiratory syncytial virus, and human metapneumovirus [7, 8]. While the development of cheaper and more user-friendly platforms will always be welcome, there needs to be a change in emphasis away from simply detecting specific organisms in a given specimen to also demonstrating that those organisms are actually causing pneumonia. The broader approach of next generation sequencing may provide novel insights into pneumonia aetiology, but has yet to show added advantage as a diagnostic tool [9].

A major recent revelation has been recognition of the lung microbiome [10]. Until recently, the lungs in health were regarded as sterile. The use of modern cultureindependent techniques has not supported this notion, consistently finding evidence of bacteria in the lower airways [10]. This important realisation, along with the increasing recognition that bacteria and viruses frequently interact in the causative pathway to pneumonia [11], has challenged our traditional paradigm of pneumonia pathogenesis. In the new paradigm, dominant species emerge from the lung ecosystem in pneumonia through uncertain mechanisms, polymicrobial pneumonia may be common [12], and the bacterial versus viral pneumonia concept is naïve and unsophisticated. There is still much to understand, but there is a clear indication that new diagnostic tools for pneumonia should not just be focused on detection of specific known pathogens.

No major new innovative approaches to pneumonia diagnostics have appeared since 2009. The potential for breath analysis has still not been realised and is the subject of ongoing investigation by many research groups $[13,14]$. There have also been no major new breakthroughs in the biomarker world to aid the aetiologic diagnosis of pneumonia. While there is some evidence that antibiotic management of pneumonia may be guided by blood procalcitonin levels [15], it is unclear whether procalcitonin is functioning as a marker for bacterial pneumonia or as a marker of disease severity. This is particularly pertinent as we move away from the simplistic bacterial versus viral pneumonia paradigm. It is possible that the answer may lie with certain combinations of protein biomarkers that may have greater utility for determining microbial aetiology in pneumonia [16], or that biomarkers are better used for purposes other than for determining the cause of pneumonia [17].

The findings of one of the world's largest pneumonia aetiology studies will be published in 2016 with great anticipation. The Pneumonia Etiology Research for Child Health $(\mathrm{PERCH})$ project is a seven-country case-control study, which is focused on the causes of severe pneumonia in young children from developing countries [18]. There is great interest in PERCH's experience deploying state of the art diagnostics at each study site. However, one of the most novel aspects of PERCH is the use of sophisticated statistical modelling techniques to help interpret diagnostic test results. Partially latent class models have been designed for estimating the population aetiology distribution and the individual aetiology probabilities for specific pneumonia pathogens [19]. This approach endeavours to overcome the known limitations of diagnostic testing, and has never before been so extensively applied to the aetiology of an infectious disease. It remains to be seen what reception this approach will receive from the scientific community.

For the diagnostic laboratory, there have been only modest changes in the approach to determining the causative pathogens of pneumonia over recent years. However, we have entered a new age that could well increase the pace of development. By opening our minds to new paradigms of pneumonia pathogenesis and increasing our understanding of the lung microbiome in health and disease, additional diagnostic tools are likely to appear that will help better guide the management of pneumonia.

\section{Competing interests}

The author declares no competing interests.

\section{Author contributions}

DM met ICMJE authorship criteria. DM contributed entirely to the drafting and writing of the paper. DM critically reviewed the manuscript for important intellectual content. DM agrees with the manuscript's results and conclusions.

\section{Funding}

The authors have no funding to report.

Received: 13 January 2016 Accepted: 22 January 2016

Published online: 12 April 2016

\section{References}

1. Murdoch DR, O'Brien KL, Scott JA, Karron RA, Bhat N, Driscoll AJ, et al. Breathing new life into pneumonia diagnostics. J Clin Microbiol. 2009;47: 3405-8. PMID:19741073, http://dx.doi.org/10.1128/JCM.01685-09.

2. Schreckenberger PC, MCAdam AJ. Point-Counterpoint: Large multiplex PCR panels should be first-line tests for detection of respiratory and intestinal pathogens. J Clin Microbiol. 2015;53:3110-5. PMID:25762770, http://dx.doi. org/10.1128/JCM.00382-15.

3. World Health Organization. Automated real-time nucleic acid amplification technology for rapid and simultaneous detection of tuberculosis and rifampicin resistance: Xpert MTB/RIF assay for the diagnosis of pulmonary and extrapulmonary TB in adults and children: policy update. Geneva: World Health Organization; 2013. 
4. Albrich WC, Madhi SA, Adrian PV, van Niekerk N, Mareletsi T, Cutland C, et al. Use of a rapid test of pneumococcal colonization density to diagnose pneumococcal pneumonia. Clin Infect Dis. 2012;54:601-9. PMID:22156852, http://dx.doi.org/10.1093/cid/cir859.

5. Tempia S, Wolter N, Cohen C, Walaza S, von Mollendorf C, Cohen AL, et al. Assessing the impact of pneumococcal conjugate vaccines on invasive pneumococcal disease using polymerase chain reaction-based surveillance: an experience from South Africa. BMC Infect Dis. 2015;15:450. PMID: 26496761, http://dx.doi.org/10.1186/s12879-015-1198-z.

6. Murdoch DR, Podmore RG, Anderson TP, Barratt K, Maze MJ, French KE, et al. Impact of routine systematic polymerase chain reaction testing on case finding for Legionnaires' disease: a pre-post comparison study. Clin Infect Dis. 2013;57: 1275-81. PMID:23899682, http://dx.doi.org/10.1093/cid/cit504.

7. Hammitt LL, Kazungu S, Morpeth SC, Gibson DG, Mvera B, Brent AJ, et al. A preliminary study of pneumonia etiology among hospitalized children in Kenya. Clin Infect Dis. 2012;54 Suppl 2:S190-9. PMID:22403235, http://dx.doi. org/10.1093/cid/cir1071.

8. Rhedin S, Lindstrand A, Hjelmgren A, Ryd-Rinder M, Öhrmalm L, Tolfvenstam T, et al. Respiratory viruses associated with community-acquired pneumonia in children: matched case-control study. Thorax. 2015;70:847-53. PMID:26077969, http://dx.doi.org/10.1136/thoraxjnl-2015-206933

9. Thorburn F, Bennett S, Modha S, Murdoch D, Gunson R, Murcia PR. The use of next generation sequencing in the diagnosis and typing of respiratory infections. J Clin Virol. 2015;69:96-100. PMID:26209388.

10. Dickson RP, Huffnagle GB. The lung microbiome: new principles for respiratory bacteriology in health and disease. PLoS Pathog. 2015;11:e1004923. PMID: 26158874, http://dx.doi.org/10.1371/journal.ppat.1004923.

11. McCullers JA. The co-pathogenesis of influenza viruses with bacteria in the lung. Nat Rev Microbiol. 2014;12:252-62. PMID:24590244, http://dx.doi.org/ 10.1038/nrmicro3231.

12. Cillóniz C, Civljak R, Nicolini A, Torres A. Polymicrobial community-acquired pneumonia: An emerging entity. Respirology. 2016;21:65-75. PMID: 26494527, http://dx.doi.org/10.1111/resp.12663.

13. van der Schee MP, Paff T, Brinkman P, van Aalderen WM, Haarman EG, Sterk PJ. Breathomics in lung disease. Chest. 2015;147:224-31. PMID:25560860, http://dx.doi.org/10.1378/chest.14-0781.

14. Wilson AD. Advances in electronic-nose technologies for the detection of volatile biomarker metabolites in the human breath. Metabolites. 2015;5: 140-63. PMID:25738426, http://dx.doi.org/10.3390/metabo5010140.

15. Markanday A. Acute phase reactants in infections: evidence-based review and a guide for clinicians. Open Forum Infect Dis. 2015;2:ofv098. http://dx. doi.org/10.1093/ofid/ofv098. PMID:26258155.

16. Huang $\mathrm{H}$, Ideh RC, Gitau E, Thézénas ML, Jallow M, Ebruke B, et al. Discovery and validation of biomarkers to guide clinical management of pneumonia in African children. Clin Infect Dis. 2014;58:1707-15. PMID:24696240.

17. Erdman LK, D'Acremont V, Hayford K, Rajwans N, Kilowoko M, Kyungu E, et al. Biomarkers of host response predict primary end-point radiological pneumonia in Tanzanian children with clinical pneumonia: a prospective cohort study. PLoS One. 2015;10:e0137592. PMID:26366571.

18. Levine OS, O'Brien KL, Deloria-Knoll M, Murdoch DR, Feikin DR, DeLuca AN, et al. The Pneumonia Etiology Research for Child Health Project: a 21st century childhood pneumonia etiology study. Clin Infect Dis. 2012;54 Suppl 2:S93-101. PMID:22403238, http://dx.doi.org/10.1093/cid/cir1052.

19. Wu Z, Deloria-Knoll M, Hammitt LL, Zeger SL. Partially latent class models for case-control studies of childhood pneumonia aetiology. J R Stat Soc Ser C Appl Stat. 2016;65:97-114. http://dx.doi.org/10.1111/rssc.12101.

\section{Submit your next manuscript to BioMed Central and we will help you at every step:}

- We accept pre-submission inquiries

- Our selector tool helps you to find the most relevant journal

- We provide round the clock customer support

- Convenient online submission

- Thorough peer review

- Inclusion in PubMed and all major indexing services

- Maximum visibility for your research

Submit your manuscript at www.biomedcentral.com/submit
Biomed Central 